\title{
Texture evolution in selective laser melted maraging stainless steel CX with martensitic transformation
}

Hadi Pirgazi ${ }^{1,2^{*}}$, Mehdi Sanjari ${ }^{3,4}$, Saeed Tamimi ${ }^{5}$, Babak Shalchi Amirkhiz ${ }^{4,3}$, Leo A.I. Kestens ${ }^{1,2}$ and

\author{
Mohsen Mohammadi ${ }^{3}$
}

\begin{abstract}
${ }^{1}$ Department of Electromechanical, Systems \& Metal Engineering, Ghent University, Ghent, Belgium 2 Department of Materials Science and Engineering, Delft University of Technology, Delft, The Netherlands

${ }^{3}$ Marine Additive Manufacturing Centre of Excellence (MAMCE), University of New Brunswick, Fredericton, NB, Canada

${ }^{4}$ CanmetMATERIALS, Natural Resources Canada, 183 Longwood Road South, Hamilton, ON, Canada L8P OA5

${ }^{5}$ Advanced Forming Research Centre (AFRC), University of Strathclyde, Glasgow, UK
\end{abstract}

\section{ABSTRACT}

Due to high local cooling rates and non-equilibrium directional solidification conditions, selective laser melting (SLM) processed metals exhibit microstructural and textural features significantly different from the conventionally processed ones. The evolution of crystallographic orientations in a maraging stainless steel (commercially known as stainless steel $(X)$ sample fabricated by the SLM process was studied through experimental and modelling approaches Electron backscattering diffraction analysis showed that the dominant texture components in martensite and austenite phases are $<111>||$ building direction and $<011>$ | | building direction, respectively. Texture simulation indicated that the formation of crystallographic orientations in the studied sample is the result of two consecutive phase transformations, from initially solidified delta ferrite phase with dominant cube fiber texture to austenite and austenite to martensite.

Keywords: Maraging steels; Martensitic phase transformation; Texture; Simulation; Selective laser melting (SLM).

\footnotetext{
* Corresponding author: Email: hadi.pirgazi@ugent.be; Phone: +32 93310449.
} 


\section{Introduction}

Selective laser melting (SLM) is a commonly employed laser powder-bed fusion based additive manufacturing (AM) technique for fabricating metal components from feedstock powder [1]. SLM uses a computer controlled high energy laser as the heat source, which layer by layer melts and fuses selective regions of the metallic powder and produces fully dense near netshape components. The main focus of most of the related research activities on SLM and other AM processes has been on the feasibility of the process for manufacturing flawless components, the resulting mechanical properties, and some limited microstructural characterizations [2-5].

AM processes have not yet entered the market as a mature and fully adopted technology. One of the open processing questions that requires further investigation pertains to the evolution of crystallographic texture during the SLM process. Deep understanding of the evolved texture is of crucial importance because: (i) many of the material properties are texture dependent [6-10]; and (ii) the crystallographic texture influences different phenomena such as recrystallization, phase transformation, and grains response to deformation during metals processing [11-13]. Due to high local cooling rates and nonequilibrium solidification conditions, metallic components produced by the SLM process represent microstructural and textural features, which significantly differ from those usually observed in metals and alloys processed by the conventional techniques (e.g. casting, forging, and extrusion).

Of particular interest in this work is a maraging stainless steel with chemical composition similar to the family of $13.8 \mathrm{Mo}$ PH stainless steels. As this type of maraging stainless steel contains a fully martensitic microstructure, it experiences a phase transformation during cooling to room temperature. The effect of phase transformation on the texture evolution of steels produced by conventional thermo-mechanical processing techniques (especially rolling) has been extensively studied [14-17]. Generally, during an allotropic phase transformation an orientation relationship (OR) between the crystal lattices of the parent and the product phases is developed. For example, the Kurdjumov and Sachs (K-S) OR [18], in which $\{111\}_{\gamma}||\{110\}_{\alpha^{\prime}}$ and $\left.\langle 110\rangle_{\gamma}||<111\right\rangle_{\alpha^{\prime}}$, was observed between lath martensite and retained austenite grains in conventionally heat-treated maraging steels [19]. It has been 
shown that phase transformations and their corresponding OR can significantly affect the final texture $[20,21]$. However, this effect has not been adequately addressed for SLM processed steels. Therefore, the main objective of this communication is to study the texture evolution in a maraging stainless steel sample fabricated by the SLM process featuring phase transformation, and to assess the source of the evolving crystallographic orientations.

\section{Materials and methods}

A gas-atomized powder of maraging stainless steel (commercially known as stainless steel CX (SS CX)) containing spherical shape particles with average size of approximately $37.5 \mu \mathrm{m}$ was used as the feedstock powder for the SLM process. The SS CX is an iron-based high strength corrosion resistant powder with a nominal chemical composition of $\mathrm{Fe}-12 \% \mathrm{Cr}-9.2 \% \mathrm{Ni}$ 1.4\%Mo-1.6\%Al-0.4\%Mn-0.4\%Si-0.05\%C (in wt.\%). More details about this feedstock powder can be found in [22]. The SLM process was carried out using an EOS M290 machine to produce a rod-shaped sample with $120 \mathrm{~mm}$ height and $12 \mathrm{~mm}$ diameter in the horizontal direction, i.e. the longitudinal direction (or the rod axis) of the printed rod was perpendicular to the building direction. To protect the melt pool and to avoid the pick-up of interstitial impurities such as oxygen, the SLM process was performed in an inert atmosphere $\left(\mathrm{Ar}-0.1 \% \mathrm{O}_{2}\right)$. Table 1 summarizes the SLM process parameters. The laser power, scanning speed, hatching distance, and powder layer thickness values were adjusted to allow for fabricating samples with the least porosity and the best mechanical properties. The hatching strategy for the SLM process was the well-known stripe scanning with $67^{\circ}$ rotation of the stripes between consecutive layers.

For the purpose of microstructural analyses, the printed sample was sectioned parallel to the longitudinal direction of the rod. For simplicity, the three orthogonal directions of the printed samples i.e. Iongitudinal direction, building direction, and transverse direction are abbreviated as LD, BD and TD, respectively. The sample was mounted in epoxy resin and polished using a Tegramin-30 Struers auto-grinder/polisher. After polishing, the sample was subjected to cleaning with an ultrasonic cleaner for 60 seconds. Electron backscattering diffraction (EBSD) technique was employed to characterize the evolved microstructure and texture in the SLM processed alloy. The EBSD measurement was carried out using a HR-FEGSEM (high-resolution field emission gun scanning electron microscope) of type FEI QUANTA 
450. To obtain statistically relevant crystallographic information, a large EBSD scan $(400 \times 425$ $\mu \mathrm{m}^{2}$ ) with a step size of $0.2 \mu \mathrm{m}$ was collected. To ensure a detailed characterization of the fine austenite grains, a smaller EBSD map $\left(100 \times 100 \mu \mathrm{m}^{2}\right)$ but with higher resolution of 50 $\mathrm{nm}$ was also recorded from the center of the previous scan. The collected orientation datasets were then post-processed using the TSL-OIM software to derive the microstructural state variables and crystallographic orientations. The average confidence index $(\mathrm{Cl})$ of the raw data equaled 0.6; however, only the orientations with $\mathrm{Cl}>0.1$ were included in subsequent texture analysis. This filtering resulted in more than $4.3 \times 10^{6}$ and $1.8 \times 10^{5}$ data points with the average $\mathrm{Cl}$ of 0.66 and 0.47 for the bcc and fcc phases, respectively. The harmonic series expansion method with a truncation of $\mathrm{L}=16$ and a Gaussian-Half-Width of $5^{\circ}$ was used to calculate the ODF and to plot the corresponding pole figures, whereby no sample symmetry was assumed.

Table 1. SLM process parameters used in this study

\begin{tabular}{|c|c|c|c|c|}
\hline Laser type & $\begin{array}{c}\text { Laser power } \\
(\mathrm{W})\end{array}$ & $\begin{array}{c}\text { Scanning speed } \\
(\mathrm{mm} / \mathrm{s})\end{array}$ & $\begin{array}{c}\text { Hatching distance } \\
(\mu \mathrm{m})\end{array}$ & $\begin{array}{c}\text { Layer thickness } \\
(\mu \mathrm{m})\end{array}$ \\
\hline Yb-Fiber & 258.7 & 1066.7 & 100 & 30 \\
\hline
\end{tabular}

\section{Results and discussion}

The building direction inverse pole figure (BD-IPF) map in Figs. $1 \mathrm{a}$ and $\mathrm{b}$ represent the microstructure of the maraging stainless steel sample fabricated by SLM. It features a typical SLM microstructure, i.e. aggregation of overlapped melt pools containing large columnar and small equiaxed grains. The formation of these different grain morphologies has been attributed to the local variations of the solidification parameters, i.e. temperature gradient (G) and growth rate (R) [23] as well as re-heating through multiple laser passes [24]. In general, a high $\mathrm{G} / \mathrm{R}$ ratio (as it is the case for the SLM process) results in the formation of a columnar grain structure [25]. As reported in similar studies [24, 26], these columnar grains are aligned with the building direction, which is antiparallel to the overall heat extraction direction from the melt pools. On the other hand, the heterogeneous nucleation at the fusion line between the previously solidified layer and the molten metal promotes the formation of small equiaxed grains, which are mostly located at the melt pool boundaries, as highlighted in the region between two yellow curves in Fig. 1a. 
A detailed description of the governing mechanisms for the microstructural evolution during the SLM process can be found elsewhere $[2,24]$. The high-resolution phase map shown in Fig. $1 c$ indicates that the microstructure consists of $96 \%$ bcc phase (i.e. martensite or $\alpha^{\prime}$ ) and $4 \%$ fcc phase (i.e. retained austenite or $\gamma$ ). The austenite phase is seen as very fine grains distributed mostly between the equiaxed martensitic grains at the vicinity of the melt pool boundaries and to less extent between columnar grains.
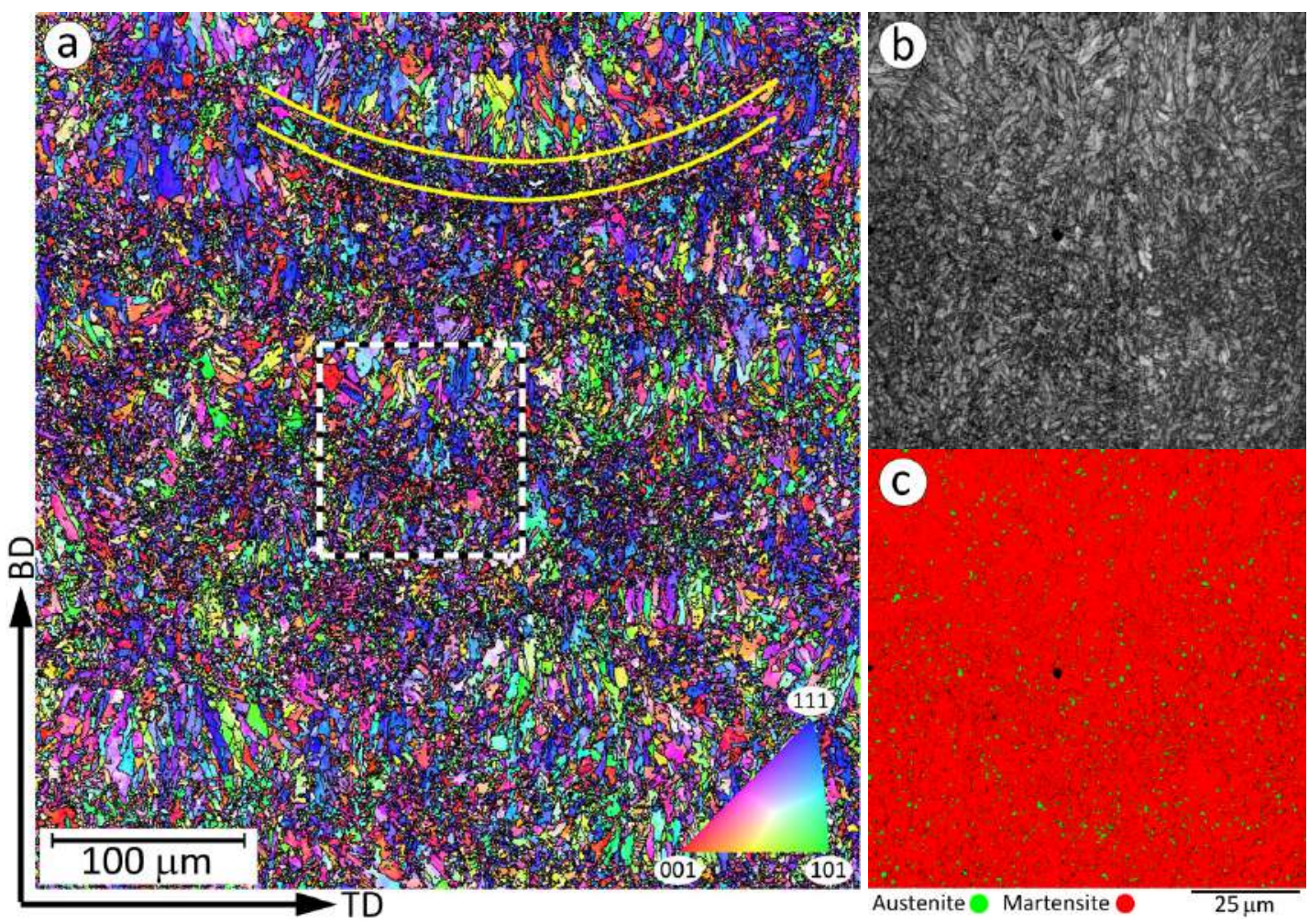

Figure 1. (a) BD-IPF map representing the microstructure of the SLM processed maraging steel sample, the yellow curves mark a melt pool boundary covered by small equiaxed grains. (b) Image quality map, and (c) phase map corresponding to the area marked by the dashed line square in Fig. 1a. The black pixels in the phase map comprise the low $\mathrm{Cl}(<0.1)$ orientations.

The presence of the $\gamma$ phase at room temperature is due to incomplete $\gamma$ to $\alpha^{\prime}$ phase transformation caused by e.g. local stabilization of the $\gamma$ phase as a result of the segregation of the alloying elements during the solidification process. Therefore, the orientation relationship (OR) across the $\mathrm{bcc} / \mathrm{fcc}$ phase boundaries is expected to correspond with the common OR types in steels (cf. Table 2). As shown in Fig. 2, the OR between adjacent $\alpha^{\prime}$ and $\gamma$ grains are distributed around the Bain circles formed by the variants of the ORs presented 
in Table 2 (except the Bain OR). A detailed analysis showed that $\sim 50 \%$ of the boundaries between fcc and bcc phases fall within $4^{\circ}$ angular deviation with respect to the Bain circle. From the phase boundaries across the Bain circle, $81 \%, 55 \%$ and $26 \%$ follow the Greninger and Troiano (G-T) [27], Nishiyama-Wassermann (N-W) [28, 29], and Pitsch [30] orientation relationships, respectively (cf. Fig. 2). The presence of an OR between parent and product phases is beneficial for understanding the evolution of crystallographic texture in the SLM processed maraging steel, as will be discussed shortly.

Table 2. Plane and direction parallelism conditions between fcc and bcc phases for different theoretical orientation relationships in steels.

\begin{tabular}{|l|l|l|}
\hline Name & \multicolumn{2}{|c|}{ Orientation relationship } \\
\hline Bain [31] & $\{100\}_{\gamma} \|\{100\}_{\alpha}$ & $<100>_{\gamma} \|<100>_{\alpha}$ \\
\hline Kurdjumov -Sachs (K-S) [18] & $\{111\}_{\gamma} \|\{110\}_{\alpha}$ & $<110>_{\gamma} \|<111>_{\alpha}$ \\
\hline Nishiyama-Wassermann (N-W) [28, 29] & $\{111\}_{\gamma} \|\{110\}_{\alpha}$ & $<112>_{\gamma} \|<111>_{\alpha}$ \\
\hline Greninger and Troiano (G-T) [27] & $\{111\}_{\gamma} \|\{110\}_{\alpha}$ & $<123>_{\gamma} \|<133>_{\alpha}$ \\
\hline Greninger and Troiano' (G-T') [27] & $\{110\}_{\gamma} \|\{111\}_{\alpha}$ & $<133>_{\gamma} \|<123>_{\alpha}$ \\
\hline Pitsch [30] & $\{100\}_{\gamma} \|\{110\}_{\alpha}$ & $<110>_{\gamma} \|<111>_{\alpha}$ \\
\hline
\end{tabular}

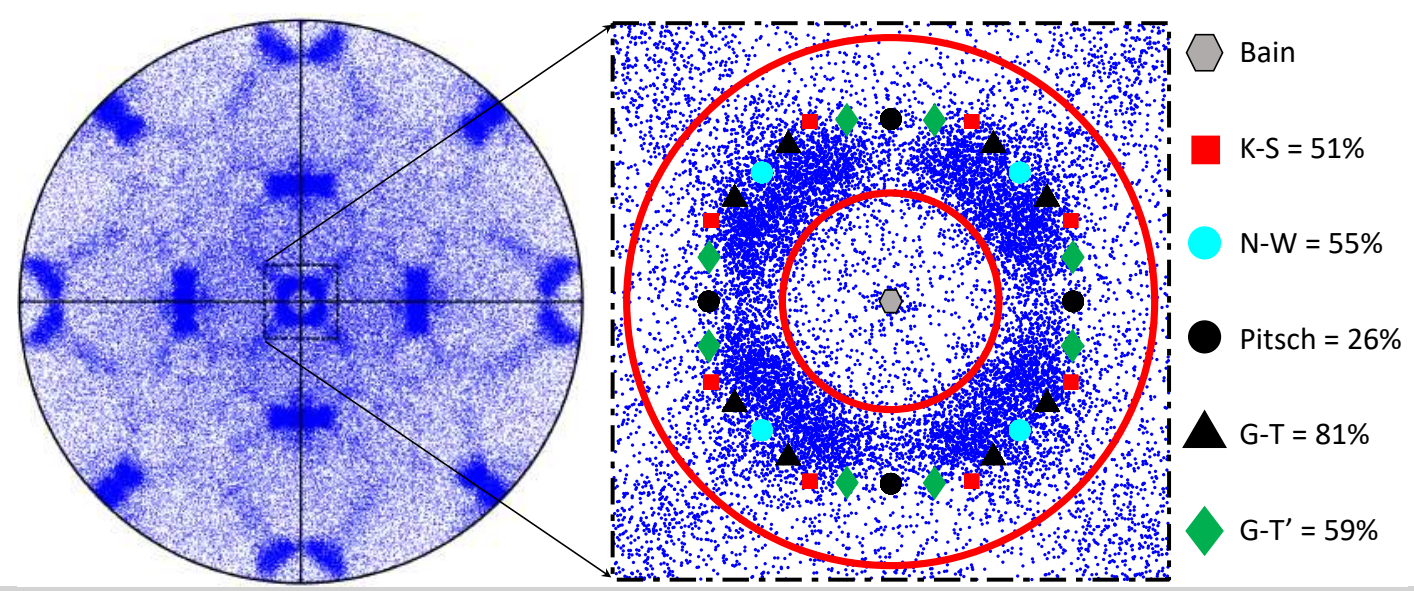

Figure 2. $\{001\}$ pole figure of the actual orientation relationships across the phase boundaries. The enlarged view of the pole figure represents the variants of different theoretical orientation relationships around the central Bain circle. The red circles mark the area with $4^{\circ}$ angular deviation with respect to the Bain circle which contains $\sim 50 \%$ of all phase boundaries. 
As shown in the pole figures (PFs) of Fig. 3, martensite and austenite phases exhibit weak textures both featuring an approximate axial sample symmetry around the building direction (i.e. the center of the PFs) with the maximum intensity at $<111>$ and $<011>$ poles, respectively. Compared to the martensite phase, the axial symmetry of the austenite phase (cf. Fig. 3a) is less ideal, which is likely related to the considerably less number of data points in this phase. The appearance of the axial symmetry is a result of (i) the directional solidification in which the preferred solidification direction (i.e. $<001>$ in bcc and fcc crystals [32]) is parallel to the direction of heat flow, and (ii) the rotation of the laser scanning direction between the consecutive layers. A fibrous texture is the most common texture that has been observed in SLM processed alloys $[24,33]$. It has been reported that the resulting texture is influenced by the melt pools geometry, which is governed by the generated temperature gradients within the melt pools. Accordingly, the melt pool geometry and the resulting crystallographic texture can be altered by changing the laser scanning parameters and/or the hatching strategy [3437]. Consequently, different solidification textures such as $<001>|| B D[24,38],<011>|| B D$ [37] and $<111>||$ BD $[39,40]$ have been reported in SLM processed metals with cubic crystal structures."

The observed textures in this study can be explained by considering the occurrence of a phase transformation in this alloy. Based on the governing OR, the martensitic phase transformation results in different crystallographic orientations. Furthermore, a fast cooling rate (as it holds for the SLM process), reduces the difference in the phase transformation driving force for different variants of the $\gamma \rightarrow \alpha^{\prime}$ phase transformation [41]. The latter results in the formation of variants with equal statistical probability and eventually reduces the overall texture intensity [42]. The occurrence of martensitic transformation without variant selection in this alloy justifies the observed weak texture (cf. Fig. 3) as opposed to SLM processed alloys, which do not experience any phase transformation (e.g. aluminum alloys [43]). 

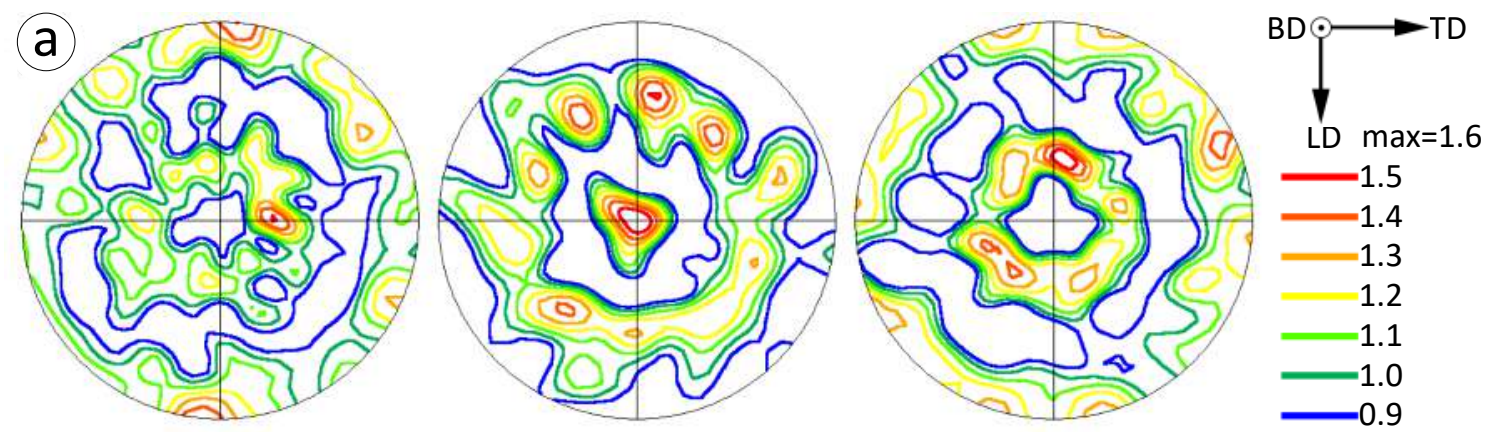

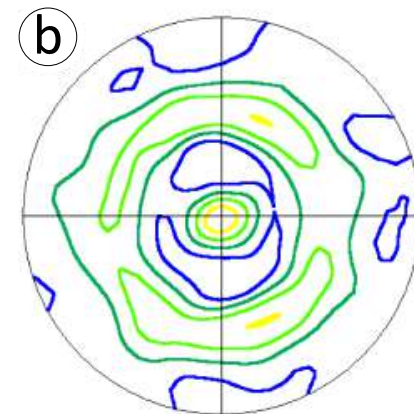

001

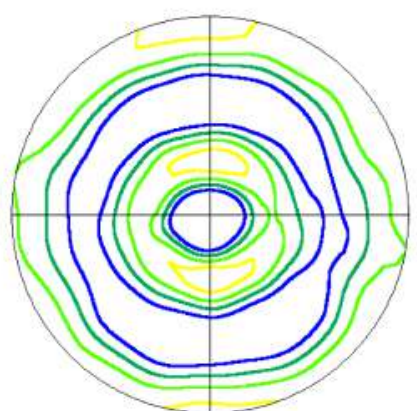

011

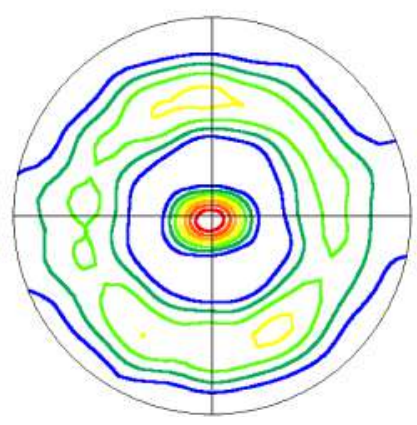

111

Figure 3. Experimental pole figures representing the crystallographic texture of (a) retained austenite and (b) martensite grains. LD, TD and BD stand for longitudinal, transverse and building direction, respectively.

Texture evolution of the austenite phase can be explained by taking into account the evolution of phases during solidification. The phase diagram of the studied alloy was calculated using the Thermo-Calc software. As Fig. 4a shows, the first phase formed upon solidification is a bcc phase (i.e. $\delta$-ferrite), which further transforms into fcc (i.e. austenite phase) and eventually into a bcc phase (i.e. martensite). The fraction of these phases as a function of temperature has been plotted in Fig. $4 \mathrm{~b}$. Around $1440^{\circ} \mathrm{C}$ the structure of the sample is composed of $13 \%$ liquid and $87 \% \delta$-ferrite phases. It should be noted that the diagrams of Fig. 4 are based on equilibrium condition, which differ from the solidification conditions of the SLM process featuring a very fast cooling rate. An increased cooling rate is expected to decrease the volume fraction of the $\delta$-ferrite phase at the expense of the austenite phase $[44,45]$. However, it can still be assumed that a high fraction of the melt pools is first solidified as the $\delta$-ferrite phase. The occurrence of two consecutive phase transformations i.e. $\delta$-ferrite $\rightarrow$ austenite $\rightarrow$ martensite during cooling, results in the observed experimental textures in this alloy. 

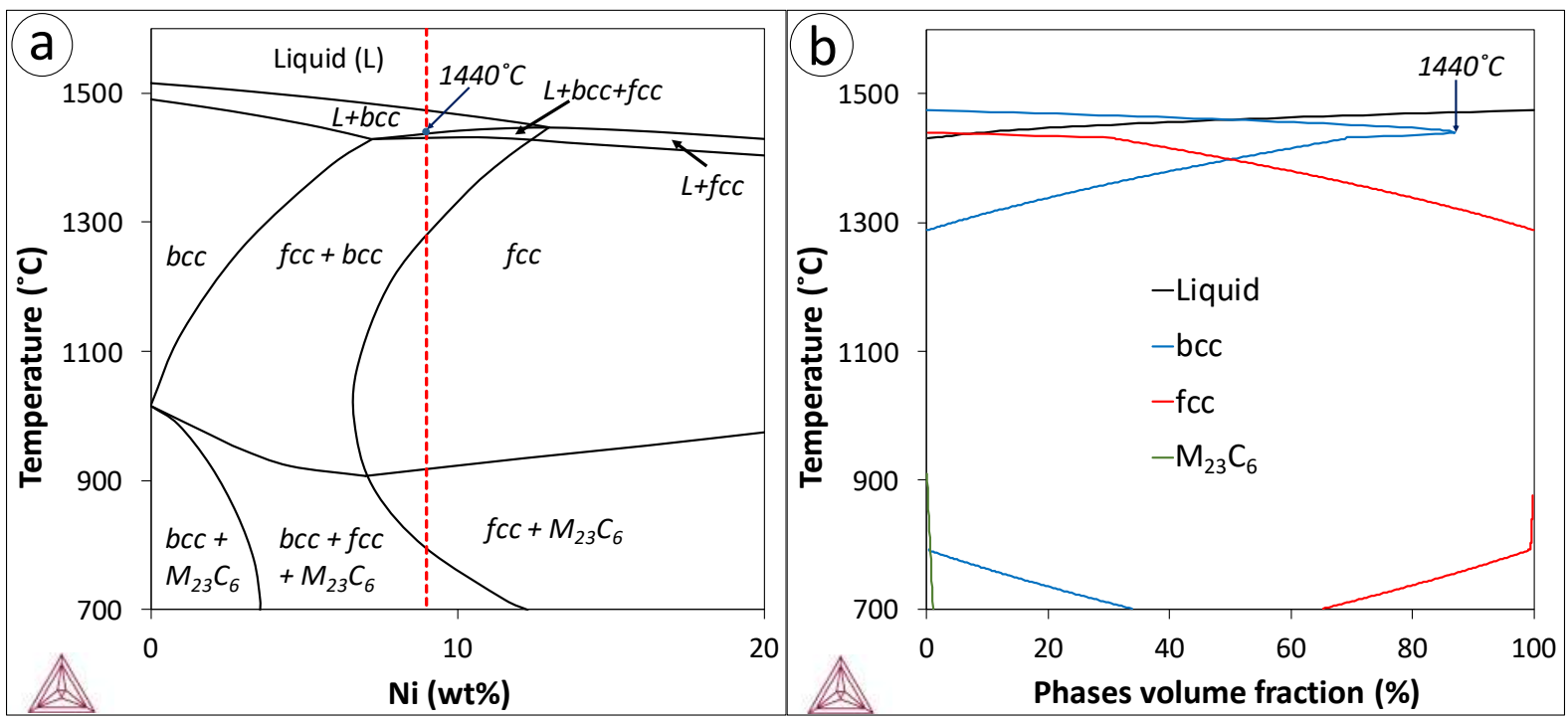

Figure 4. (a) Fe-Ni phase diagram and (b) fraction of phases during solidification and subsequent cooling, calculated using the Thermo-Calc software. The dashed red line in (a) corresponds to the chemical composition of the studied alloy.

To better understand the mechanism of texture evolution in the SLM-SS CX, a simple model including two consecutive transformations, i.e. $\delta \rightarrow \gamma \rightarrow \alpha^{\prime}$, based on the G-T OR was utilized to model the texture of austenite and martensite phases. The authenticity of the OR for $\delta$ to $\gamma$ transformation has been validated by several studies including Haghdadi et al. [46] and Xu et al. [44]. To simplify the model, $\delta$-ferrite is assumed to be the only phase directly solidified from the melt. Consequently, the effect of the directly solidified austenite is neglected because due to a low volume fraction ( $13 \%)$, its influence is insignificant. The texture of the $\delta$-ferrite as the first solidified phase is assumed to be $a<111>|| B D$ texture. This assumption is validated by (i) the reported $<111>|| B D$ texture in the literature where similar laser parameters and hatching strategy have been employed, e.g. $[39,40,47]$, and (ii) the geometry of melt pool. The secondary electron image presented in Fig. 5 shows the melt pool boundaries in the BD-TD section. As the laser scanning direction (SD) rotates $67^{\circ}$ between the layers, only when SD is perpendicular to the plane of observation the distance between two neighboring melt pools equals the hatching distance (i.e. $100 \mu \mathrm{m}$ in this case), and the melt pools geometry can be assessed correctly. For example, in the melt pools marked by the dashed lines in Fig. 5b, SD is perpendicular to the plane of observation. The largest temperature gradient in a melt pool lies along the direction perpendicular to the melt pool boundary [34]. In this study, the average angle $(\omega)$ between heat flow directions (i.e. red arrows in Fig. $5 b)$ and the negative $B D$ equates $\sim 42^{\circ}$. The same value can be roughly 
considered for the average angle between the heat flow direction and the negative BD in the longitudinal cross section. As explained by Thijs et al. [47] these two rotations around the SD and TD result in a $<111>$ almost parallel to $B D$ texture. Accordingly, a set of 1000 random orientations with the $\left\langle 111>\right.$ direction aligned within $30^{\circ}$ of the building direction was generated as the texture of the $\delta$-ferrite phase (cf. Fig. 6a).
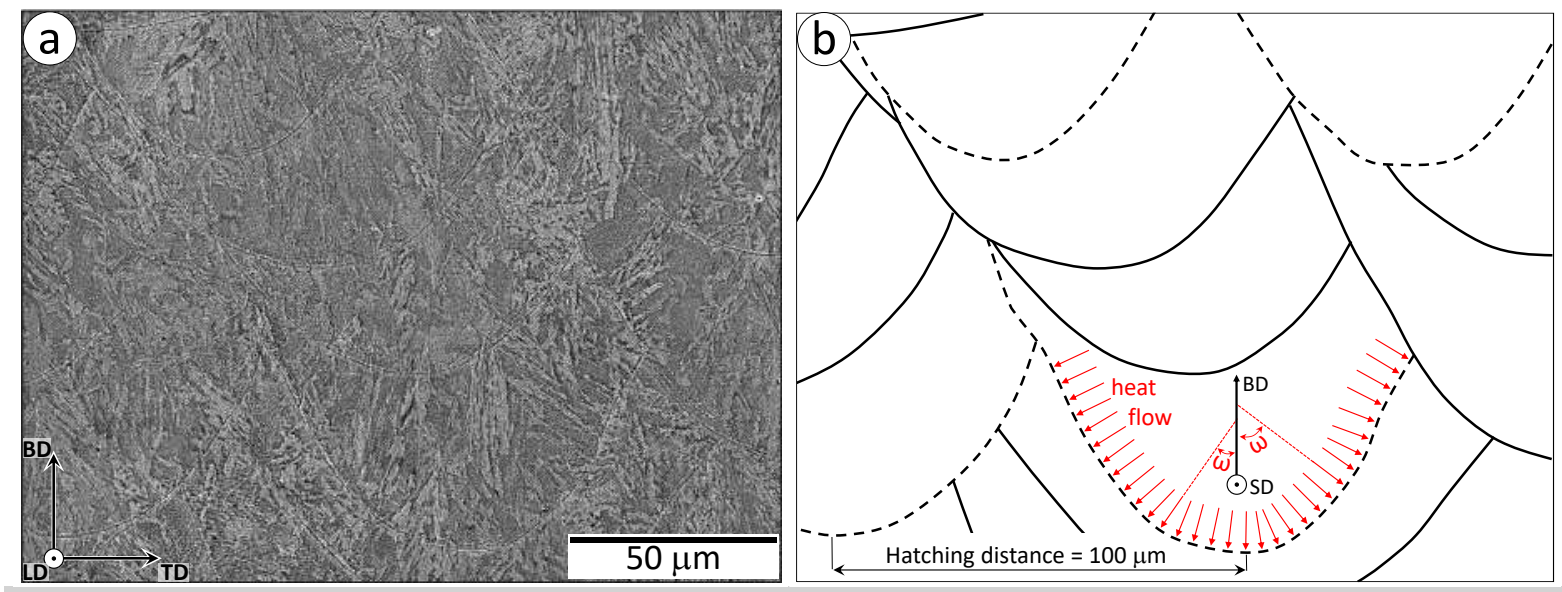

Figure 5. (a) Secondary electron image, and (b) the corresponding melt pool boundaries in the BD-TD cross section.

To simulate the texture of the austenite phase, the G-T OR was applied on the initial $\delta$-ferrite texture. Assuming that $\mathrm{T}_{\mathrm{GT}}$ represents the transformation matrix for the $\mathrm{fcc} \rightarrow \mathrm{bcc}$ phase transformation based on the G-T OR, the following equation was employed:

$$
g_{\gamma i}=T_{G T}^{-1}\left(O_{i} g_{\delta}\right)
$$

where $O_{i}$ is the crystal symmetry operator, $\mathrm{g}_{\gamma}$ and $\mathrm{g}_{\delta}$ are the orientations of the austenite and delta ferrite phases, respectively. $\mathrm{T}^{-1} \mathrm{GT}$ represents the inverse of the transformation matrix for $\delta \rightarrow \gamma$ phase transformation. To this purpose, it was assumed that each ferrite orientation can transform into six randomly selected austenite variants (out of 24 possible variants associated with the G-T OR). This simplifying assumption was necessary as the physical origin of variant selection, and consequently, the number of variants in this alloy and the current processing condition is not well-understood. On the other hand, the fast cooling rate associated with the SLM process gives the same priority to all variants to be selected. The simulated texture of the austenite phase (cf. Fig. $6 \mathrm{~b}$ exhibits the expected axial symmetry with 
a maximum intensity at $\langle 011>|| B D$, which is in general agreement with the experimental results (i.e. Fig. 3a).

In a similar way, the texture of the martensite phase was simulated by employing the G-T OR and transforming each austenite orientation resulting from the previous step (i.e. 6000 orientations) into six randomly selected martensitic variants, employing the following equation:

$$
g_{\alpha^{\prime} i}=T_{G T}\left(O_{i} g_{\gamma}\right)
$$

where $g_{\alpha^{\prime}}$ is the orientation of the martensite phase and $T_{G T}$ represents the transformation matrix for the $\gamma \rightarrow \alpha^{\prime}$ phase transformation based on the G-T OR.

As Fig. 6c shows, there is clearly a good compatibility between simulated texture of martensite and the experimental result presented in Fig. 3b. Both sets of PFs (i.e. Fig. 3b and Fig. 6 c) show a close to ideal axial symmetry with a maximum intensity at $<111>|| B D$ and the second maximum at $\angle 001>|| B D$. It should be noted that the phase transformations were applied based on the G-T OR, as it was found to be the dominant OR between the fcc and bcc phases in this alloy. However, our analysis showed that the choice of the OR (e.g. N-W, K-S, Pitsch or $\mathrm{GT}^{\prime}$ ) does not significantly change the modelled textures. This is probably due to the small angular deviation between these ORs, the random choice of variants, and the approximated initial texture which eventually resulted in similar modelled textures.

The presented results indicate that the occurrence of two consecutive phase transformations determines the final texture observed in the studied sample. The melt pools are initially solidified mostly as $\delta$-ferrite with a dominant $<111>$ ||BD texture. The occurrence of $\delta \rightarrow \gamma$ phase transformations results in the formation of a $\angle 011>|| B D$ texture. The second transformation (i.e. $\gamma \rightarrow \alpha^{\prime}$ ) gives rise to the evolution of a weak texture with $<111>|| B D$ as the dominant texture fiber. The similarities between the crystallographic textures observed in the bcc phases (i.e. $\delta$ and $\alpha^{\prime}$ ) after the double transformation is an indication for some degree of texture memory effect. An ideal texture memory effect is considered to appear when the initial texture is completely recovered after the double phase transformation. It has been reported that this effect arises from the occurrence of a specific variant selection mechanism during phase transformations, which favors nucleation at parent phase grain 
boundaries that allow for a double K-S (or other) reference correspondence [48, 49]. Therefore, the absence of variant selection in this alloy -as discussed earlier- weakens the texture memory effect, resulting in a considerably lower intensity texture in the $\alpha^{\prime}$ phase in comparison to the $\delta$ phase.

The fast cooling rate during the SLM process can affect the fraction of initially solidified phases and the fraction of phases after each transformation step. Furthermore, the alternating heating and cooling cycles associated with the deposition of next layers can affect the thermal equilibrium condition of the phases and consequently the resulting textures. To determine the effect of these parameters on the evolution of the texture during the SLM process, more detailed studies are underway. 

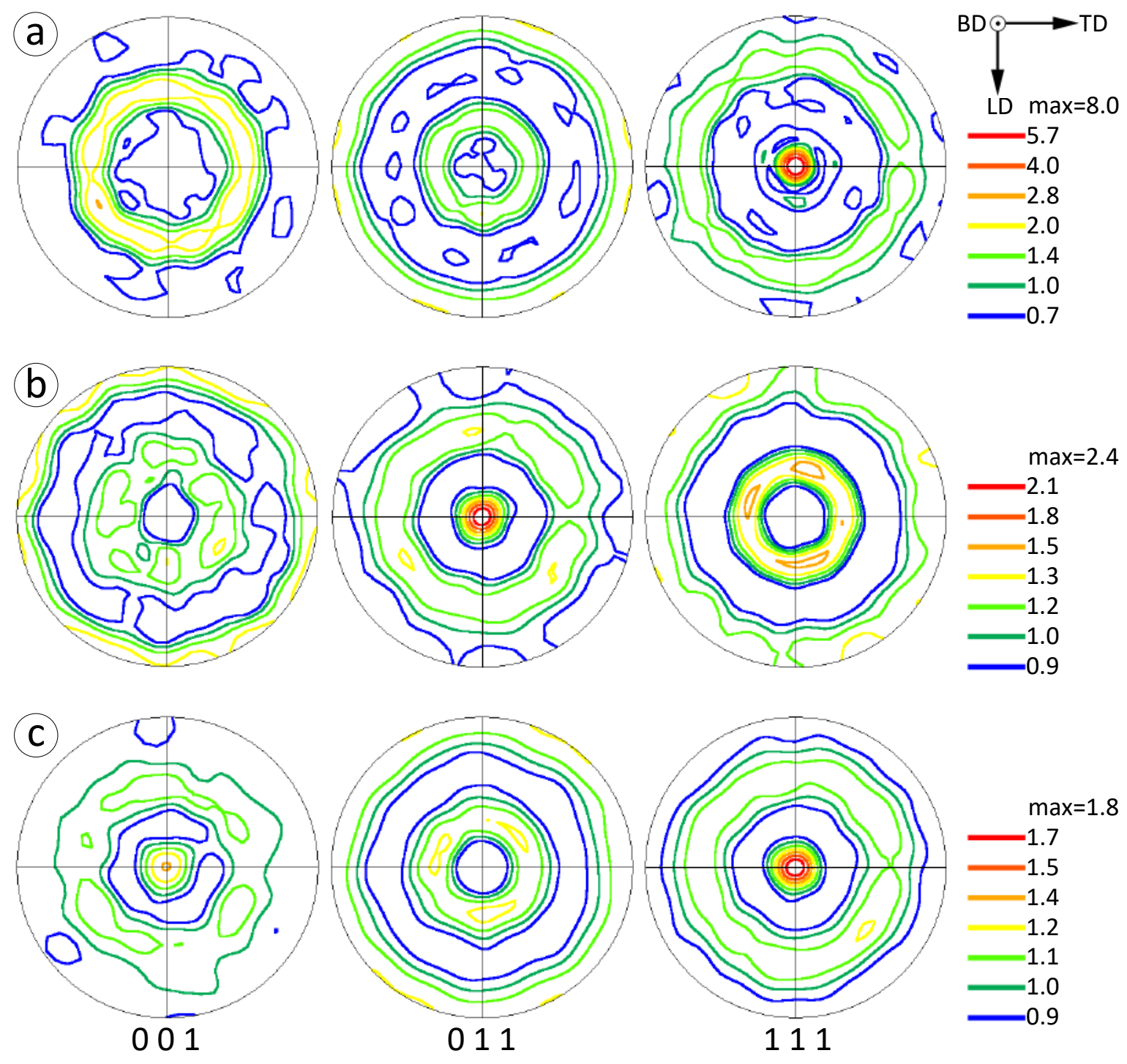

Figure 6. Simulated pole figures representing the crystallographic texture of (a) $\delta$-ferrite, (b) austenite, and (c) martensite phase after transformation based on the G-T orientation relationship.

\section{Conclusions}

In summary, the SLM processed maraging stainless steel sample in this study shows a microstructure mostly composed of martensitic grains and a small fraction of retained austenite. The dominant texture components in martensite and austenite phases was found to be $<111>|| B D$ and $<011>|| B D$, respectively. A model for texture simulation was developed by taking into consideration the following assumptions: (i) $\delta$-ferrite as the first solidified phase has a dominant $<111>|| B D$ texture, and (ii) $\delta$-ferrite to austenite and austenite to martensite phase transformations occur according to the Greninger and Troiano orientation relationship 
with a random selection of the variants. The simulated textures correctly matched the experimental ones leading to the conclusion that the texture formation in the SLM processed maraging stainless steel (stainless steel $C X$ ) is the result of the occurrence of the two consecutive phase transformations.

\section{Acknowledgments}

This work was supported by the Natural Sciences and Engineering Research Council of Canada (NSERC) [grant number RGPIN-2016-04221], New Brunswick Innovation Foundation (NBIF) [grant number RIF2017-071], Atlantic Canada Opportunities Agency (ACOA)-Atlantic Innovation Fund (AIF) [project number 210414]; and Mitacs Accelerate Program [grant number IT10669].

\section{Data availability}

The raw/processed data required to reproduce these findings cannot be shared at this time as the data also forms part of an ongoing study.

\section{Compliance with ethical standards}

Conflict of interest The authors declare that they have no conflict of interest. 


\section{References}

1. Collins PC, Brice DA, Samimi P, et al (2016) Microstructural Control of Additively Manufactured Metallic Materials. Annu Rev Mater Res 46:63-91. https://doi.org/10.1146/annurev-matsci-070115-031816

2. Sanjari M, Hadadzadeh A, Shahriairi A, et al (2020) On the Effect of Building Direction on the Microstructure and Grain Morphology of a Selective Laser Melted Maraging Stainless Steel. In: TMS 2020 149th Annual Meeting \& Exhibition Supplemental Proceedings. Springer International Publishing, Cham, pp 285-295

3. Asgari H, Baxter C, Hosseinkhani K, Mohammadi M (2017) On microstructure and mechanical properties of additively manufactured AlSi10Mg_200C using recycled powder. Mater Sci Eng A 707:148-158. https://doi.org/10.1016/j.msea.2017.09.041

4. Tallon J, Cyr E, Lloyd A, Mohammadi M (2020) Crush performance of additively manufactured maraging steel microlattice reinforced plates. Eng Fail Anal 108:104231. https://doi.org/https://doi.org/10.1016/j.engfailanal.2019.104231

5. Sanjari M, Hadadzadeh A, Pirgazi $H$, et al (2020) Selective laser melted stainless steel CX: Role of built orientation on microstructure and micro-mechanical properties. Mater Sci Eng A 786:139365. https://doi.org/https://doi.org/10.1016/j.msea.2020.139365

6. Pirgazi $\mathrm{H}$, Akbarzadeh $\mathrm{A}$ (2008) Characterization of nanostructured aluminum sheets processed by accumulative roll bonding. Int J Mod Phys B 22:2840-2847. https://doi.org/10.1142/S0217979208047663

7. Naghdy S, Pirgazi H, Verleysen P, et al (2017) Morphological and crystallographic anisotropy of severely deformed commercially pure aluminium by three-dimensional electron backscatter diffraction. J Appl Crystallogr 50:1512-1523. https://doi.org/https://doi.org/10.1107/S1600576717012754

8. Pirgazi H, Glowinski K, Morawiec A, Kestens LAI (2015) Three-dimensional characterization of grain boundaries in pure nickel by serial sectioning via mechanical polishing. J Appl Crystallogr 48:1672-1678.

https://doi.org/10.1107/S1600576715017616

9. Pirgazi $H$, Petrov R, Kestens L (2012) Modeling the magnetic properties of nonoriented electrical steels based on microstructural parameters. In: Materials Science Forum. pp 734-737

10. Pirgazi H, Petrov RH, Kestens LAl (2016) Effect of Grain Boundary-Magnetic Domain Interaction on the Magnetization Behavior of Non-Oriented Electrical Steels. Steel Res Int 87:210-218. https://doi.org/10.1002/srin.201400608

11. Basak A, Das S (2015) Epitaxy and Microstructure Evolution in Metal Additive Manufacturing. Annu Rev Mater Res 46:annurev-matsci-070115-031728. https://doi.org/10.1146/annurev-matsci-070115-031728

12. Gäumann M, Henry S, Cléton F, et al (1999) Epitaxial laser metal forming: analysis of microstructure formation. Mater Sci Eng A 271:232-241.

https://doi.org/10.1016/S0921-5093(99)00202-6

13. Takata N, Nishida R, Suzuki A, et al (2018) Crystallographic features of microstructure in maraging steel fabricated by selective laser melting. Metals (Basel) 8:440. 
https://doi.org/10.3390/met8060440

14. Kestens LAI, Pirgazi H (2016) Texture formation in metal alloys with cubic crystal structures. Mater Sci Technol 32:1303-1315.

https://doi.org/10.1080/02670836.2016.1231746

15. Emadoddin E, Akbarzadeh A, Petrov R, et al (2011) Influence of cold-rolling reduction on retained austenite texture in cold-rolled and intercritically annealed TRIP-assisted steel. J Appl Crystallogr 44:1190-1197. https://doi.org/10.1107/S0021889811041069

16. Jonas JJ (2009) Transformation Textures Associated with Steel Processing. In: Haldar A, Suwas S, Bhattacharjee D (eds) Microstructure and Texture in Steels: and Other Materials. Springer London, London, pp 3-17

17. Hutchinson WB, Ryde L, Bate PS (2005) Transformation Textures in Steels. Mater Sci Forum 495-497:1141-1150. https://doi.org/10.4028/www.scientific.net/MSF.495497.1141

18. Kurdjumow G, Sachs G (1930) Über den Mechanismus der Stahlhärtung. Zeitschrift für Phys 64:325-343. https://doi.org/10.1007/BF01397346

19. Morito S, Huang X, Furuhara T, et al (2006) The morphology and crystallography of lath martensite in alloy steels. Acta Mater 54:5323-5331.

https://doi.org/https://doi.org/10.1016/j.actamat.2006.07.009

20. Butron-Guillén MP, Jonas JJ (1996) Effect of Finishing Temperature on Hot Band Textures in an IF Steel. Isij Int 36:68-73.

https://doi.org/https://doi.org/10.2355/isijinternational.36.68

21. Kestens L, Verbeken K, Jonas J (2001) Orientation selection by nucleation and growth in highly strained low carbon steels. Recryst GRAIN GROWTH, VOLS 12 1-2:695-706

22. Asgari $\mathrm{H}$, Mohammadi M (2018) Microstructure and mechanical properties of stainless steel CX manufactured by Direct Metal Laser Sintering. Mater Sci Eng A 709:82-89. https://doi.org/10.1016/j.msea.2017.10.045

23. David SA, Vitek JM (1989) Correlation between solidification parameters and weld microstructures. Int Mater Rev 34:213-245.

https://doi.org/10.1179/imr.1989.34.1.213

24. Hadadzadeh A, Amirkhiz BS, Li J, Mohammadi M (2018) Columnar to equiaxed transition during direct metal laser sintering of AlSi10Mg alloy: Effect of building direction. Addit Manuf 23:121-131. https://doi.org/10.1016/j.addma.2018.08.001

25. Yan F, Xiong W, Faierson EJ (2017) Grain Structure Control of Additively Manufactured Metallic Materials. Materials (Basel) 10:. https://doi.org/10.3390/ma10111260

26. Hadadzadeh A, Amirkhiz BS, Shakerin S, et al (2020) Microstructural investigation and mechanical behavior of a two-material component fabricated through selective laser melting of AlSi10Mg on an Al-Cu-Ni-Fe-Mg cast alloy substrate. Addit Manuf 31:100937. https://doi.org/https://doi.org/10.1016/j.addma.2019.100937

27. Greninger AB, Troiano AR (1949) The mechanism of martensite formation. Trans AIME 185:590-598

28. Nishiyama Z (1934) X-ray investigation of the mechanism of the transformation from face centered cubic lattice to body centered cubic. Sci Rep Tohoku Univ 23:637

29. Wassermann G (1935) Ueber den Mechanismus der [alpha]-[gamma]-Umwandlung des Eisens. Mitt K-Wilh-Inst Eisenforsch 17:149-155

30. Pitsch W (1962) Der Orientierungszusammenhang zwischen Zementit und Austenit. Acta Metall 10:897-900. https://doi.org/https://doi.org/10.1016/0001- 


\section{0(62)90108-6}

31. Bain EC, Dunkirk NY (1924) The nature of martensite. Trans Am Inst Mining, Metall Pet Eng 70:25. https://doi.org/10.1007/BF02642442

32. Kou S (2003) Welding Metallurgy. John Wiley \& Sons, Ltd

33. Thijs L, Kempen K, Kruth J-P, Humbeeck] J [Van (2013) Fine-structured aluminium products with controllable texture by selective laser melting of pre-alloyed AlSi10Mg powder. Acta Mater 61:1809-1819.

https://doi.org/https://doi.org/10.1016/j.actamat.2012.11.052

34. Sun S-H, Hagihara K, Nakano T (2018) Effect of scanning strategy on texture formation in Ni-25at.\%Mo alloys fabricated by selective laser melting. Mater Des 140:307-316. https://doi.org/https://doi.org/10.1016/j.matdes.2017.11.060

35. Andreau O, Koutiri I, Peyre P, et al (2019) Texture control of 316L parts by modulation of the melt pool morphology in selective laser melting. J Mater Process Technol 264:21-31. https://doi.org/https://doi.org/10.1016/j.jmatprotec.2018.08.049

36. Sun Z, Tan X, Tor SB, Chua CK (2018) Simultaneously enhanced strength and ductility for 3D-printed stainless steel 316L by selective laser melting. NPG Asia Mater 10:127136. https://doi.org/10.1038/s41427-018-0018-5

37. Jadhav SD, Dadbakhsh S, Goossens L, et al (2019) Influence of selective laser melting process parameters on texture evolution in pure copper. J Mater Process Technol 270:47-58. https://doi.org/https://doi.org/10.1016/j.jmatprotec.2019.02.022

38. Ghoncheh MH, Sanjari M, Cyr E, et al (2020) On the solidification characteristics, deformation, and functionally graded interfaces in additively manufactured hybrid aluminum alloys. Int J Plast 133:102840.

https://doi.org/https://doi.org/10.1016/j.ijplas.2020.102840

39. OH J, ISHIMOTO T, SUN S, NAKANO T (2019) Crystallographic Texture Formation of Pure Tantalum by Selective Laser Melting Method. J Smart Process 8:151-154. https://doi.org/10.7791/jspmee.8.151

40. Jones DR, Fensin SJ, Ndefru BG, et al (2018) Spall fracture in additive manufactured tantalum. J Appl Phys 124:225902. https://doi.org/10.1063/1.5063930

41. Farabi E, Hodgson PD, Rohrer GS, Beladi H (2018) Five-parameter intervariant boundary characterization of martensite in commercially pure titanium. Acta Mater 154:147-160. https://doi.org/https://doi.org/10.1016/j.actamat.2018.05.023

42. Beladi H, Chao Q, Rohrer GS (2014) Variant selection and intervariant crystallographic planes distribution in martensite in a Ti-6Al-4V alloy. Acta Mater 80:478-489. https://doi.org/https://doi.org/10.1016/j.actamat.2014.06.064

43. Liu X, Zhao C, Zhou X, et al (2019) Microstructure of selective laser melted AlSi10Mg alloy. Mater Des 168:107677. https://doi.org/https://doi.org/10.1016/j.matdes.2019.107677

44. Xu PG, Yin F, Nagai K (2006) Solidification cooling rate and as-cast textures of lowcarbon steel strips. Mater Sci Eng A 441:157-166. https://doi.org/https://doi.org/10.1016/j.msea.2006.09.001

45. Xu PG, Yin FX, Nagai K (2006) Effect of Cooling Rate on As-Cast Texture of Low-Carbon Steel Strips During Rapid Solidification. In: Advanced Structural and Functional Materials Design. Trans Tech Publications Ltd, pp 41-48

46. Haghdadi N, Cizek P, Hodgson PD, et al (2018) Effect of ferrite-to-austenite phase transformation path on the interface crystallographic character distributions in a duplex stainless steel. Acta Mater 145:196-209. 
https://doi.org/https://doi.org/10.1016/j.actamat.2017.11.057

47. Thijs L, Montero Sistiaga ML, Wauthle R, et al (2013) Strong morphological and crystallographic texture and resulting yield strength anisotropy in selective laser melted tantalum. Acta Mater 61:4657-4668.

https://doi.org/https://doi.org/10.1016/j.actamat.2013.04.036

48. Yoshinaga $\mathrm{N}$, Inoue $\mathrm{H}$, Kawasaki $\mathrm{K}$, et al (2007) Factors Affecting Texture Memory Appearing through \&alpha;\&rarr;\&gamma;\&rarr;\&alpha; Transformation in IF Steels. Mater Trans 48:2036-2042. https://doi.org/10.2320/matertrans.MA200704

49. Hutchinson B, Kestens LAI (2008) Origins of Texture Memory in Steels. In: Applications of Texture Analysis. John Wiley \& Sons, Ltd, pp 281-290 


\section{Figure Captions}

Figure 1. (a) BD-IPF map representing the microstructure of the SLM processed maraging steel sample, the yellow curves mark a melt pool boundary covered by small equiaxed grains. (b) Image quality map, and (c) phase map corresponding to the area marked by the dashed line square in Fig. 1a. The black pixels in the phase map comprise the low $\mathrm{Cl}(<0.1)$ orientations.

Figure 2. $\{001\}$ pole figure of the actual orientation relationships across the phase boundaries. The enlarged view of the pole figure represents the variants of different theoretical orientation relationships around the central Bain circle. The red circles mark the area with $4^{\circ}$ angular deviation with respect to the Bain circle which contains $\sim 50 \%$ of all phase boundaries.

Figure 3. Experimental pole figures representing the crystallographic texture of (a) retained austenite and (b) martensite grains. LD, TD and BD stand for longitudinal, transverse and building direction, respectively.

Figure 4. (a) Fe-Ni phase diagram and (b) fraction of phases during solidification and subsequent cooling, calculated using the Thermo-Calc software. The dashed red line in (a) corresponds to the chemical composition of the studied alloy.

Figure 5. (a) Secondary electron image, and (b) the corresponding melt pool boundaries in the BD-TD cross section.

Figure 6. Simulated pole figures representing the crystallographic texture of (a) $\delta$-ferrite, (b) austenite, and (c) martensite phase after transformation based on the G-T orientation relationship.

\section{Table Captions}

Table 1. SLM process parameters used in this study

Table 2. Plane and direction parallelism conditions between fcc and bcc phases for different theoretical orientation relationships in steels. 